International Journal of Applied Mathematics

Volume 32 No. $4 \quad 2019,641-661$

ISSN: 1311-1728 (printed version); ISSN: 1314-8060 (on-line version)

doi: http://dx.doi.org/10.12732/ijam.v32i4.8

\title{
COMPLETE INTERSECTION CALABI-YAU TEN-FOLDS
}

\author{
Vladislav Dumachev \\ Department of Mathematics \\ Voronezh Institute of the Ministry \\ of Internal Affairs of Russia \\ Voronezh - 394065, RUSSIA
}

\begin{abstract}
In this paper a complete intersection Calabi-Yau 10-folds are considered. Their Hodge diamond, Todd classes and Chern characters for sheaves of differential $k$-forms are calculated.
\end{abstract}

AMS Subject Classification: 14J32, 14J40, 14Q10

Key Words: Calabi-Yau manifolds, Hodge diamond, Chern character

\section{Introduction}

All definitions in this article are conventional, as in [1]. We work in projective space $\mathbb{P}^{n}$ over an algebraically closed field of arbitrary characteristic. Let $x$ be divisor degree of hypersurface $X \in \mathbb{P}^{n}$. We denote $X_{m}=\bigcap_{i=1}^{k} S_{i}^{s_{i}} \in \mathbb{P}^{n}$ as $m$-fold, which is a complete intersection of $k$ hypersurfaces $S_{i}$ degree $s_{i}$. Then $X$ is a Calabi-Yau if $x=\sum s_{i}=n+1$. For a sheaf of differential forms $\Omega_{X}^{i}=\Lambda^{i} \Omega_{X}$ we introduce Hodge numbers $h^{i j}=\operatorname{dim} H^{\mathrm{i}}\left(\Omega_{\mathrm{X}}^{\mathrm{j}}\right)$, which not only are symmetrical: $h^{i j}=h^{j i}$, but Serre symmetrical also: $h^{i j}=h^{n-i, n-j}$. If we have Hodge numbers then Betti numbers may be calculated as

$$
b_{k}=\sum_{i+j=k} h^{i j} .
$$

Received: June 17, 2019

(C) 2019 Academic Publications 
We can also define the Euler characteristic of $X$ as the alternating sum of the Betti numbers:

$$
\chi(X)=\sum_{k}(-)^{k} b_{k}
$$

For clarity, we rotate the matrix $h^{i j}$ on $45^{\circ}$ and call it as the Hodge diamond. So, for $n=\operatorname{dim} X_{n}$, we will have:

$$
\begin{aligned}
& h^{0,0} \quad b_{0}=h^{0,0} \\
& h^{1,0} \quad h^{0,1} \quad b_{1}=h^{1,0}+h^{0,1}
\end{aligned}
$$

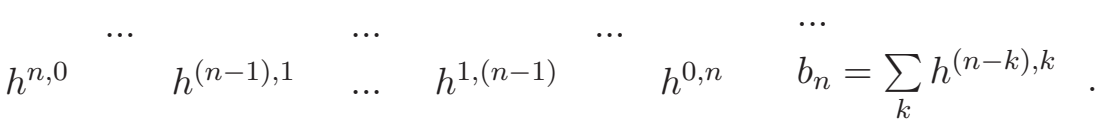

$$
\begin{aligned}
& \begin{array}{llll}
h^{n,(n-1)} & h^{(n-1), 3} & & \cdots \\
& & \cdots
\end{array} \\
& h^{n, n} \quad b_{2 n}=h^{n, n}
\end{aligned}
$$

If $X=\bigcap_{k=1}^{t} S_{k}$ is complete intersection of t-hypersurfaces of degree $s_{k}$, then $N_{X}=\bigoplus_{k=1}^{t} \mathcal{O}_{X}\left(-s_{k}\right)$ and the sheaf $\mathcal{O}_{X}$ can be determined with used the following recurrence relations:

$$
\begin{gathered}
0 \rightarrow \mathcal{O}_{S_{1} \cap S_{2} \cap \ldots \cap S_{k-1}}\left(-s_{k}\right) \rightarrow \mathcal{O}_{S_{1} \cap S_{2} \cap \ldots \cap S_{k-1}} \rightarrow \mathcal{O}_{S_{1} \cap S_{2} \cap \ldots \cap S_{k}} \rightarrow 0 \\
0 \rightarrow \mathcal{O}_{S_{1} \cap S_{2} \cap \ldots \cap S_{k-2}}\left(-s_{k-1}\right) \rightarrow \mathcal{O}_{S_{1} \cap S_{2} \cap \ldots \cap S_{k-2}} \rightarrow \mathcal{O}_{S_{1} \cap S_{2} \cap \ldots \cap S_{k-1}} \rightarrow 0 \\
\quad \ldots \\
0 \rightarrow \mathcal{O}_{S_{1}}\left(-s_{2}\right) \rightarrow \mathcal{O}_{S_{1}} \rightarrow \mathcal{O}_{S_{1} \cap S_{2}} \rightarrow 0 \\
0 \rightarrow \mathcal{O}_{\mathbb{P} n}\left(-s_{1}\right) \rightarrow \mathcal{O}_{\mathbb{P} n} \rightarrow \mathcal{O}_{S_{1}} \rightarrow 0
\end{gathered}
$$

Now, for calculations of $H^{i}\left(\Omega_{X}^{k}\right)$ we can get the power series

$$
0 \rightarrow \operatorname{Sym}^{k} N_{X} \rightarrow \operatorname{Sym}^{k-1} N_{X} \otimes \Omega_{\mathbb{P}^{n} \mid X} \rightarrow \ldots \rightarrow \Omega_{\mathbb{P}^{n} \mid X}^{k} \rightarrow \Omega_{X}^{k} \rightarrow 0 .
$$

\section{Todd and Chern classes}

As shown in [2] for any rational complete intersection Calabi-Yau $X$ all non-zero entries of the Hodge diamond are always lying on its equator or on the central column. Also, $h^{i i}=1$ if $i \neq m / 2$. Therefore, we can simplify all calculation with use of characteristic classes theory. 
We take the Riemann-Roch-Hirzebruch equation

$$
\chi(E, X)=\int_{X} \operatorname{ch}(\mathrm{E}) \wedge \operatorname{td}\left(\mathrm{T}_{\mathrm{X}}\right),
$$

attach it to $E=\bigwedge^{q} \Omega_{X}=\Omega_{X}^{q}$ and rewrite over Chern classes of tangent bundle $c\left(T_{X}\right)=\sum_{i} c_{i}\left(T_{X}\right)=\prod_{i}\left(1+\alpha_{i}\right)$ :

$$
\begin{gathered}
{ }^{i} \mathrm{Td}\left(\mathrm{T}_{\mathrm{X}}\right)=\prod_{\mathrm{i}} \frac{\alpha_{\mathrm{i}}}{1-\mathrm{e}^{-\alpha_{\mathrm{i}}}}=\sum \mathrm{td}_{\mathrm{i}} \\
\mathrm{Ch}\left(\Omega_{X}^{q}\right)=\sum_{i_{1}<i_{2}<\ldots<i_{q}} e^{\alpha_{i_{1}}} \ldots e^{\alpha_{i_{q}}}=\sum \operatorname{ch}_{\mathrm{i}} .
\end{gathered}
$$

Here $\alpha_{i}$ are the Chern roots of $T_{\mathbb{P} n}$.

For the first time the classes $\operatorname{td}\left(\mathrm{T}_{\mathrm{X}}\right)$ up to $\mathrm{td}_{6}$ were obtained in [3] (p.14). In the original paper [4] (p.221) Eq.(61) coincides with the $\mathrm{td}_{6}$ in our notation. All classes up to $\mathrm{td}_{9}$ were obtained in [5], up to $\mathrm{td}_{10}$ in [2].

The Taylor series for $(2)$ is

$$
\operatorname{ch}_{\mathrm{m}}\left(\Omega_{n}^{p}\right)=\sum_{s=1}^{\# I} A_{I_{s}} X^{I_{s}}
$$

where according to $[11]$

$$
A_{I_{s}}=\frac{1}{I_{s} !}\left(\begin{array}{l}
n-\# I_{s} \\
p-\# I_{s}
\end{array}\right), \quad X^{I_{s}}=\sum_{k}^{n} \bigwedge^{\# I_{s}} x_{k}^{I_{s}} .
$$

The multi-index

$$
I=\{m,(m-1,1),(m-2,2), \ldots,(2, \underbrace{1, \ldots, 1}_{m-2}),(\underbrace{1,1, \ldots, 1}_{m})\}
$$

is a tuple of a partition of integer $m$ and $\# I$ is length of a tuple $I$.

For Chern characters of $n$-folds $X$ from (2) we have the following theorems.

\section{Theorem 1.}

$$
\begin{aligned}
\operatorname{ch}_{5}\left(\Omega_{n}^{p}\right) & =\frac{(-1)^{m}}{5 !}\left[\left(\begin{array}{c}
n-1 \\
p-1
\end{array}\right) c_{1}^{5}+\left[\left(\begin{array}{c}
n-2 \\
p-2
\end{array}\right)-\left(\begin{array}{c}
n-1 \\
p-1
\end{array}\right)\right] 5 c_{2} c_{1}^{3}\right. \\
& +\left[\left(\begin{array}{c}
n-1 \\
p-1
\end{array}\right)-\left(\begin{array}{c}
n-2 \\
p-2
\end{array}\right)\right] 5 c_{2}^{2} c_{1} \\
& +\left[4\left(\begin{array}{c}
n-3 \\
p-3
\end{array}\right)-5\left(\begin{array}{c}
n-2 \\
p-2
\end{array}\right)+\left(\begin{array}{c}
n-1 \\
p-1
\end{array}\right)\right] 5 c_{3} c_{1}^{2} \\
& +\left[-2\left(\begin{array}{c}
n-3 \\
p-3
\end{array}\right)+3\left(\begin{array}{c}
n-2 \\
p-2
\end{array}\right)-\left(\begin{array}{c}
n-1 \\
p-1
\end{array}\right)\right] 5 c_{2} c_{3} \\
& +\left[12\left(\begin{array}{c}
n-4 \\
p-4
\end{array}\right)-22\left(\begin{array}{c}
n-3 \\
p-3
\end{array}\right)+11\left(\begin{array}{c}
n-2 \\
p-2
\end{array}\right)-\left(\begin{array}{c}
n-1 \\
p-1
\end{array}\right)\right] 5 c_{4} c_{1}
\end{aligned}
$$




$$
\left.+\left[24\left(\begin{array}{l}
n-5 \\
p-5
\end{array}\right)-60\left(\begin{array}{l}
n-4 \\
p-4
\end{array}\right)+50\left(\begin{array}{c}
n-3 \\
p-3
\end{array}\right)-15\left(\begin{array}{c}
n-2 \\
p-2
\end{array}\right)+\left(\begin{array}{c}
n-1 \\
p-1
\end{array}\right)\right] 5 c_{5}\right] .
$$

Proof. Let the multi-index (4)

$$
I=\{5,(4,1),(3,2),(3,1,1),(2,2,1),(2,1,1,1),(1,1,1,1,1)\}
$$

be a tuple of a integer partition. Since $m=5$ then we have the following expansion.

For $s=1: I_{1}=5, \# I_{1}=1$,

$$
\begin{aligned}
A_{I_{1}}=A_{5}=\frac{1}{5 !}\left(\begin{array}{c}
n-\# I_{1} \\
p-\# I_{1}
\end{array}\right)=\frac{1}{5 !}\left(\begin{array}{c}
n-1 \\
p-1
\end{array}\right), \\
X^{I_{1}}=\sum_{k}^{n} \bigwedge^{\# I_{1}} x_{k}^{I_{1}}=\sum_{k=1}^{n} x_{k}^{5} \\
=c_{1}^{5}-5 c_{2} c_{1}^{3}+5 c_{3} c_{1}^{2}+5 c_{2}^{2} c_{1}-5 c_{4} c_{1}-5 c_{2} c_{3}+5 c_{5} .
\end{aligned}
$$

For $s=2: I_{2}=(4,1), \# I_{1}=2$,

$$
\begin{aligned}
A_{I_{2}} & =A_{4,1}=\frac{1}{4 ! 1 !}\left(\begin{array}{c}
n-\# I_{2} \\
p-\# I_{2}
\end{array}\right)=\frac{1}{4 !}\left(\begin{array}{l}
n-2 \\
p-2
\end{array}\right), \\
X^{I_{2}} & =\sum_{k}^{n} \bigwedge^{\# I_{2}} x_{k}^{I_{2}}=\sum_{k_{1}<k_{2}}^{n} x_{k_{1}}^{4} x_{k_{2}}^{1} \\
& =c_{2} c_{1}^{3}-c_{3} c_{1}^{2}-3 c_{2}^{2} c_{1}+c_{4} c_{1}+5 c_{2} c_{3}-5 c_{5} .
\end{aligned}
$$

For $s=3: I_{3}=(3,2), \# I_{3}=2$,

$$
\begin{gathered}
A_{I_{3}}=A_{3,2}=\frac{1}{3 ! 2 !}\left(\begin{array}{c}
n-\# I_{3} \\
p-\# I_{3}
\end{array}\right)=\frac{1}{3 ! 2 !}\left(\begin{array}{c}
n-2 \\
p-2
\end{array}\right), \\
X^{I_{3}}=\sum_{k}^{n} \bigwedge^{\# I_{3}} x_{k}^{I_{3}}=\sum_{k_{1}<k_{2}}^{n} x_{k_{1}}^{3} x_{k_{2}}^{2}=-2 c_{3} c_{1}^{2}+c_{2}^{2} c_{1}+5 c_{4} c_{1}-c_{2} c_{3}-5 c_{5} .
\end{gathered}
$$

For $s=4: I_{4}=(3,1,1), \# I_{4}=3$,

$$
A_{I_{4}}=A_{3,1,1}=\frac{1}{3 ! 1 ! 1 !}\left(\begin{array}{c}
n-\# I_{4} \\
p-\# I_{4}
\end{array}\right)=\frac{1}{3 !}\left(\begin{array}{c}
n-3 \\
p-3
\end{array}\right),
$$




$$
X^{I_{4}}=\sum_{k}^{n} \bigwedge_{k}^{\# I_{4}} x_{k}^{I_{4}}=\sum_{k_{1}<k_{2}<k_{3}}^{n} x_{k_{1}}^{3} x_{k_{2}}^{1} x_{k_{2}}^{1}=c_{3} c_{1}^{2}-c_{4} c_{1}-2 c_{2} c_{3}+5 c_{5} .
$$

For $s=5: I_{5}=(2,2,1), \# I_{5}=3$,

$$
\begin{gathered}
A_{I_{5}}=A_{2,2,1}=\frac{1}{2 ! 2 ! 1 !}\left(\begin{array}{c}
n-\# I_{5} \\
p-\# I_{5}
\end{array}\right)=\frac{1}{4}\left(\begin{array}{c}
n-3 \\
p-3
\end{array}\right), \\
X^{I_{5}}=\sum_{k}^{n} \bigwedge^{\# I_{5}} x_{k}^{I_{5}}=\sum_{k_{1}<k_{2}<k_{3}}^{n} x_{k_{1}}^{2} x_{k_{2}}^{2} x_{k_{2}}^{1}=c_{2} c_{3}-3 c_{1} c_{4}+5 c_{5} .
\end{gathered}
$$

For $s=6: I_{6}=(2,1,1,1), \# I_{6}=4$,

$$
\begin{gathered}
A_{I_{6}}=A_{2,1,1,1}=\frac{1}{2 ! 1 ! 1 ! 1 !}\left(\begin{array}{c}
n-\# I_{6} \\
p-\# I_{6}
\end{array}\right)=\frac{1}{2 ! 1 ! 1 ! 1 !}\left(\begin{array}{c}
n-4 \\
p-4
\end{array}\right), \\
X^{I_{6}}=\sum_{k}^{n} \bigwedge^{\# I_{6}} x_{k}^{I_{6}}=\sum_{k_{1}<k_{2}<k_{3}<k_{4}}^{n} x_{k_{1}}^{2} x_{k_{2}}^{1} x_{k_{3}}^{1} x_{k_{4}}^{1}=c_{1} c_{4}-5 c_{5} .
\end{gathered}
$$

For $s=7: I_{7}=(1,1,1,1,1), \# I_{7}=5$,

$$
\begin{gathered}
A_{I_{7}}=A_{1,1,1,1,1}=\frac{1}{1 ! 1 ! 1 ! 1 ! 1 !}\left(\begin{array}{c}
n-\# I_{7} \\
p-\# I_{7}
\end{array}\right)=\left(\begin{array}{c}
n-5 \\
p-5
\end{array}\right), \\
X^{I_{7}}=\sum_{k}^{n} \bigwedge^{\# I_{7}} x_{k}^{I_{7}}=\sum_{k_{1}<k_{2}<k_{3}<k_{4}<k_{5}}^{n} x_{k_{1}}^{1} x_{k_{2}}^{1} x_{k_{3}}^{1} x_{k_{4}}^{1} x_{k_{5}}^{1}=c_{5} .
\end{gathered}
$$

Substituting this into (3), we obtain (5).

Equations from $\operatorname{ch}_{0}\left(\Omega_{n}^{p}\right)$ to $\operatorname{ch}_{4}\left(\Omega_{n}^{p}\right)$ are in work [11]. Taking into account that $h_{i j}$ is symmetric, we do not write out the highest values for $\mathrm{ch}_{i}$. The complete formulas for $\operatorname{ch}_{\mathrm{m}}\left(\Omega_{10}^{p}\right)$ are given in Appendix.

\section{Hodge diamond}

The Hodge diamond of 2-fold Calabi-Yau may be seen in [1] (p. 590), for 3-folds in [6] (p. 45). Four to nine-folds were considered in [7]-[11].

For example, we find the Hodge diamond of 10 -fold Calabi-Yau $X_{10} \in \mathbb{P}^{15}$, which is a complete intersection of two quadrics, two cubic and sextic $X_{10}=$ $S^{6} \cap S^{3} \cap S^{3} \cap S^{2} \cap S^{2}$ (Fig.1). 
Summing the Betti numbers

$$
\mathbf{b}=(1,0,1,0,1,0,1,0,1,0,6354857582,0,1,0,1,0,1,0,1,0,1),
$$

we obtain $\chi=6354857592$.

This result is easily verified using the theory of characteristic classes. For any morphism $X \stackrel{f}{\longrightarrow} \mathbb{P}^{n}$ with using the bundle

$$
0 \rightarrow T_{X} \rightarrow f^{*} T_{\mathbb{P} n} \rightarrow N_{f} \rightarrow 0,
$$

we find Chern class $c\left(T_{X}\right)$. Defining $\left.c\left(\mathcal{O}_{\mathbb{P}} n(d)\right)\right)=1+d t$, for a complete intersection $k$ hypersurfaces $X=\bigcap_{i=1}^{k} S_{i}$ of degree $s_{i}$ we obtain $c\left(N_{f}\right)=\prod_{i=1}^{k}\left(1+s_{i} t\right)$. The Euler sequence dual to (6) has the form

$$
0 \rightarrow \mathcal{O}_{\mathbb{P}^{n}} \rightarrow \mathcal{O}_{\mathbb{P}^{n}}(1)^{\oplus(n+1)} \rightarrow T_{\mathbb{P}^{n}} \rightarrow 0 .
$$

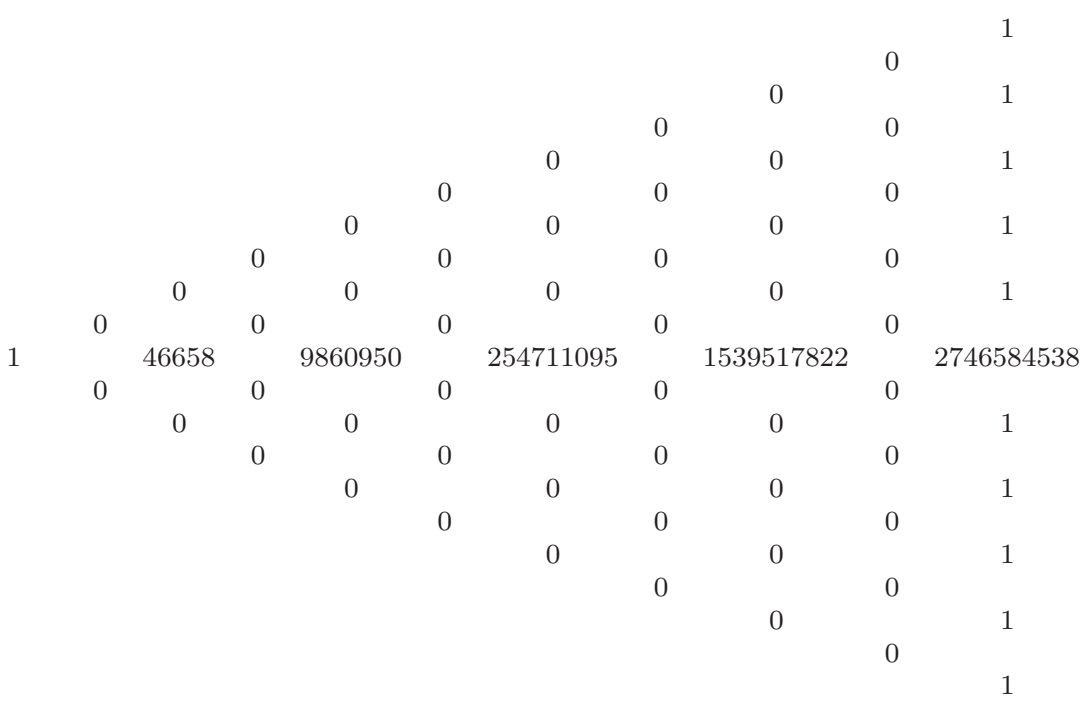

Fig 1. Half Hodge diamond for $X_{10}=S^{6} \cap S^{3} \cap S^{3} \cap S^{2} \cap S^{2} \in \mathbb{P}^{15}$

From this $c\left(T_{\mathbb{P}^{n}}\right)=(1+t)^{n+1}$, hence

$$
c\left(T_{X}\right)=\frac{(1+t)^{n+1}}{\prod_{i=1}^{k}\left(1+s_{i} t\right)} .
$$


The Euler characteristic of $m$-fold is

$$
\chi=\int_{X} c_{m}(X)=\int_{\mathbb{P}^{n}} c_{n-m}\left(N_{f}\right) \wedge c_{m}\left(T_{X}\right)=c_{n-m}\left(N_{f}\right) \cdot c_{m}\left(T_{X}\right) .
$$

For the intersection of $X_{10}=S^{6} \cap S^{3} \cap S^{3} \cap S^{2} \cap S^{2} \in \mathbb{P}^{15}$ we have

$$
\begin{aligned}
c\left(N_{f}\right)= & (1+2 t)^{2}(1+3 t)^{2}(1+6 t) \\
= & 1+16 t+\ldots+216 t^{5}, \quad \text { i.e. } \quad c_{5}\left(N_{f}\right)=216 ; \\
c\left(T_{X}\right)= & \frac{(1+t)^{16}}{(1+2 t)^{2}(1+3 t)^{2}(1+6 t)} \\
\approx & 1+23 t^{2}-90 t^{3}+\ldots+29420637 t^{10}+\ldots \\
& \text { i.e. } \quad c_{10}\left(T_{X}\right)=29420637 .
\end{aligned}
$$

Therefore the Euler characteristic from (7) has the same value $\chi$ :

$$
\chi=\int_{X} c_{10}(X)=\int_{\mathbb{P}^{15}} c_{5}\left(N_{f}\right) \wedge c_{10}\left(T_{X}\right)=6354857592 .
$$

In the following table we show the Hodge numbers of the 10-fold Calabi-Yay that are complete intersections in ordinary projective spaces. 


\begin{tabular}{rlrrrrrr}
\hline $\mathrm{N}$ & \multicolumn{1}{c}{$\mathbb{P}^{n} \mid X_{10}$} & $h^{19}+1$ & \multicolumn{1}{c}{$h^{28}+1$} & \multicolumn{1}{c}{$h^{37}+1$} & \multicolumn{1}{c}{$h^{46}+1$} & \multicolumn{1}{c}{$h^{55}$} & $\chi$ \\
\hline 1 & $11 \mid 12$ & 1351935 & 387277321 & 10501578386 & 63459767087 & 112835748610 & 261535698072 \\
2 & $12 \mid 112$ & 1058070 & 299664796 & 8111318371 & 49015898362 & 87164322830 & 202020202032 \\
3 & $12 \mid 103$ & 596544 & 162512872 & 4373000879 & 26426295482 & 47012291482 & 108937103040 \\
4 & $12 \mid 94$ & 289393 & 73627803 & 1960861422 & 11850301257 & 21096306270 & 48866466024 \\
5 & $12 \mid 85$ & 131534 & 30752880 & 808129348 & 4884193394 & 8703004804 & 20149419120 \\
6 & $12 \mid 76$ & 68770 & 15076856 & 391715181 & 2367483432 & 4222038470 & 9770726952 \\
7 & $13 \mid 1022$ & 764229 & 212652976 & 5740234801 & 34687936648 & 61696775528 & 142979952840 \\
8 & $13 \mid 932$ & 395602 & 104608116 & 2801884659 & 16932261018 & 30131946918 & 69810245712 \\
9 & $13 \mid 842$ & 176266 & 42869502 & 1133563160 & 6850811216 & 12202062940 & 28256903232 \\
10 & $13 \mid 833$ & 187380 & 46211960 & 1224444300 & 7399928290 & 13178323160 & 30519867024 \\
11 & $13 \mid 752$ & 76764 & 17040272 & 443823979 & 2682451162 & 4782826362 & 11069610720 \\
12 & $13 \mid 743$ & 77310 & 17209063 & 448520426 & 2710861649 & 4833211732 & 11186548632 \\
13 & $13 \mid 662$ & 49410 & 10496126 & 271147941 & 1638789922 & 2923763030 & 6764729832 \\
14 & $13 \mid 653$ & 35384 & 7192420 & 184451493 & 1114857104 & 1990033714 & 4603106520 \\
15 & $13 \mid 644$ & 31483 & 6272228 & 160344972 & 969186642 & 1730378210 & 4002048864 \\
16 & $13 \mid 554$ & 19289 & 3620803 & 91502942 & 553072081 & 988321166 & 2284751400 \\
17 & $14 \mid 9222$ & 502681 & 136161240 & 3660098253 & 22118092152 & 39351041592 & 91180750248 \\
18 & $14 \mid 8322$ & 235688 & 59673238 & 1587491884 & 9593761744 & 17080617640 & 39562942752 \\
19 & $14 \mid 7422$ & 95886 & 21937280 & 574296078 & 3470961362 & 6186495792 & 14321077008 \\
\hline
\end{tabular}




\begin{tabular}{rcrrrrrr}
\hline $\mathrm{N}$ & \multicolumn{1}{c}{$\mathbb{P}^{n} \mid X_{10}$} & $h^{19}+1$ & \multicolumn{1}{c}{$h^{28}+1$} & \multicolumn{1}{c}{$h^{37}+1$} & \multicolumn{1}{c}{$h^{46}+1$} & \multicolumn{1}{c}{$h^{55}$} & \multicolumn{1}{c}{ (10008 } \\
\hline 20 & $14 \mid 7332$ & 23114108 & 606066279 & 3662937364 & 6527955590 & 1511239112 \\
21 & $14 \mid 6522$ & 43039 & 8998032 & 231860759 & 1401372052 & 2500618752 & 5785166520 \\
22 & $14 \mid 6432$ & 38399 & 7881154 & 202538442 & 1224198392 & 2184839974 & 5054152752 \\
23 & $14 \mid 6333$ & 38530 & 7920660 & 203638425 & 1230856290 & 2196642090 & 5081549904 \\
24 & $14 \mid 5532$ & 22214 & 4265428 & 108246067 & 654265906 & 1168787566 & 2702386800 \\
25 & $14 \mid 5442$ & 16689 & 3071896 & 77361956 & 467607438 & 835810598 & 1931926560 \\
26 & $14 \mid 5433$ & 15534 & 2825939 & 71033730 & 429366685 & 767556980 & 1774040760 \\
27 & $14 \mid 4443$ & 9582 & 1626046 & 40313192 & 243672656 & 436068724 & 1007311680 \\
28 & $15 \mid 82222$ & 296244 & 77053898 & 2058180880 & 12437961524 & 22138399096 & 51285384192 \\
29 & $15 \mid 73222$ & 123904 & 29460438 & 775921645 & 4689373994 & 8354688906 & 19344448872 \\
30 & $15 \mid 64222$ & 46524 & 9819074 & 253534016 & 1532391356 & 2733962504 & 6325544448 \\
31 & $15 \mid 63322$ & 46658 & 9860950 & 254711095 & 1539517822 & 2746584538 & 6354857592 \\
32 & $15 \mid 55222$ & 26349 & 5198688 & 132557517 & 801191856 & 1430767376 & 3308716200 \\
33 & $15 \mid 54322$ & 18122 & 3378568 & 85305198 & 515622794 & 921448312 & 2130097680 \\
34 & $15 \mid 53332$ & 16554 & 3038336 & 76523471 & 462557228 & 826755338 & 1911026520 \\
35 & $15 \mid 44422$ & 10820 & 1874874 & 46665072 & 282062916 & 504621208 & 1165848576 \\
36 & $15 \mid 44332$ & 8889 & 1490552 & 36874020 & 222887278 & 398935958 & 921457440 \\
37 & $15 \mid 43333$ & 6805 & 1091415 & 26768310 & 161806005 & 289801110 & 669146184 \\
38 & $16 \mid 722222$ & 153384 & 37547847 & 993366254 & 6003343207 & 10692441908 & 24761263296 \\
\hline
\end{tabular}




\begin{tabular}{rlrrrrrr}
\hline $\mathrm{N}$ & \multicolumn{1}{c}{$\mathbb{P}^{n} \mid X_{10}$} & \multicolumn{1}{c}{$h^{19}+1$} & \multicolumn{1}{c}{$h^{28}+1$} & \multicolumn{1}{c}{$h^{37}+1$} & \multicolumn{1}{c}{$h^{46}+1$} & \multicolumn{1}{c}{$h^{55}$} & \multicolumn{1}{c}{$\chi$} \\
\hline 39 & $16 \mid 632222$ & 56454 & 12274403 & 318540030 & 1925259019 & 3433641176 & 7945900992 \\
40 & $16 \mid 542222$ & 21104 & 4032790 & 102276008 & 618191144 & 1104385104 & 2553427200 \\
41 & $16 \mid 533222$ & 19187 & 3604810 & 91179609 & 551140982 & 984774220 & 2276663400 \\
42 & $16 \mid 443222$ & 9820 & 1674918 & 41571352 & 251279000 & 449639832 & 1038710016 \\
43 & $16 \mid 433322$ & 7299 & 1184720 & 29127002 & 176063618 & 315276974 & 728042256 \\
44 & $16 \mid 333332$ & 4600 & 690555 & 16706445 & 100983735 & 181064910 & 417835584 \\
45 & $17 \mid 6222222$ & 68249 & 15276362 & 398310604 & 2407322932 & 4291990838 & 9933947136 \\
46 & $17 \mid 5322222$ & 22204 & 4270387 & 108471654 & 655654587 & 1171154332 & 2707992000 \\
47 & $17 \mid 4422222$ & 10784 & 1868762 & 46525568 & 281223716 & 503104192 & 1162361856 \\
48 & $17 \mid 4332222$ & 7748 & 1270210 & 31293600 & 189160892 & 338673976 & 782138880 \\
49 & $17 \mid 3333222$ & 4510 & 674400 & 16302921 & 98544816 & 176702646 & 407755944 \\
50 & $18 \mid 52222222$ & 25659 & 5051946 & 128859556 & 778877268 & 1390835778 & 3216464640 \\
51 & $18 \mid 43222222$ & 8130 & 1343098 & 33145048 & 200354436 & 358664908 & 828366336 \\
52 & $18 \mid 33322222$ & 4262 & 630265 & 15202306 & 91892629 & 164803024 & 380261952 \\
53 & $19 \mid 42_{1} \ldots 2_{8}$ & 8420 & 1398170 & 34547760 & 208837220 & 373808600 & 863391744 \\
54 & $19 \mid 332_{1} \ldots 2_{7}$ & 3819 & 552682 & 13272932 & 80231060 & 143938402 & 332059392 \\
55 & $20 \mid 32_{1} \ldots 2_{9}$ & 3140 & 437210 & 10415920 & 62961700 & 113029400 & 260665344 \\
56 & $21 \mid 2_{1} \ldots 2_{11}$ & 2180 & 281690 & 6601520 & 39903140 & 71733400 & 165310464 \\
\hline
\end{tabular}




\section{Appendix}

Taylor series coefficients for

$$
\operatorname{Ch}\left(\Omega_{10}^{p}\right)=\sum \operatorname{ch}_{i}
$$

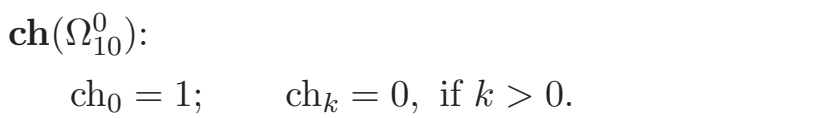

$\operatorname{ch}\left(\Omega_{10}^{1}\right)$ :

$\operatorname{ch}_{0}=10 ; \quad \operatorname{ch}_{1}=-c_{1} ; \quad \operatorname{ch}_{2}=\frac{1}{2}\left(c_{1}^{2}-2 c_{2}\right) ;$

$\mathrm{ch}_{3}=\frac{1}{3 !}\left(-c_{1}^{3}+3 c_{2} c_{1}-3 c_{3}\right)$

$\operatorname{ch}_{4}=\frac{1}{4 !}\left(c_{1}^{4}-4 c_{2} c_{1}^{2}+4 c_{3} c_{1}+2 c_{2}^{2}-4 c_{4}\right) ;$

$\operatorname{ch}_{5}=\frac{1}{5 !}\left(-c_{1}^{5}+5 c_{2} c_{1}^{3}-5 c_{3} c_{1}^{2}-5 c_{2}^{2} c_{1}+5 c_{4} c_{1}+5 c_{2} c_{3}-5 c_{5}\right) ;$

$\operatorname{ch}_{6}=\frac{1}{6 !}\left(\begin{array}{l}c_{1}^{6}-6 c_{2} c_{1}^{4}+6 c_{3} c_{1}^{3}+9 c_{2}^{2} c_{1}^{2}-6 c_{4} c_{1}^{2} \\ -12 c_{2} c_{3} c_{1}+6 c_{5} c_{1}-2 c_{2}^{3}+3 c_{3}^{2}+6 c_{2} c_{4}-6 c_{6}\end{array}\right) ;$

$\operatorname{ch}_{7}=\frac{1}{7 !}\left(\begin{array}{l}-c_{1}^{7}+7 c_{2} c_{1}^{5}-7 c_{3} c_{1}^{4}-14 c_{2}^{2} c_{1}^{3}+7 c_{4} c_{1}^{3}+21 c_{2} c_{3} c_{1}^{2} \\ -7 c_{5} c_{1}^{2}+7 c_{2}^{3} c_{1}-7 c_{3}^{2} c_{1}-14 c_{2} c_{4} c_{1} \\ +7 c_{6} c_{1}-7 c_{2}^{2} c_{3}+7 c_{3} c_{4}+7 c_{2} c_{5}-7 c_{7}\end{array}\right)$

$\operatorname{ch}_{8}=\frac{1}{8 !}\left(\begin{array}{l}c_{1}^{8}-8 c_{2} c_{1}^{6}+8 c_{3} c_{1}^{5}+20 c_{2}^{2} c_{1}^{4}-8 c_{4} c_{1}^{4}-32 c_{2} c_{3} c_{1}^{3} \\ +8 c_{5} c_{1}^{3}-16 c_{2}^{3} c_{1}^{2}+12 c_{3}^{2} c_{1}^{2}+24 c_{2} c_{4} c_{1}^{2}-8 c_{6} c_{1}^{2} \\ +24 c_{2}^{2} c_{3} c_{1}-16 c_{3} c_{4} c_{1}-16 c_{2} c_{5} c_{1}+8 c_{7} c_{1}+2 c_{2}^{4} \\ -8 c_{2} c_{3}^{2}+4 c_{4}^{2}-8 c_{2}^{2} c_{4}+8 c_{3} c_{5}+8 c_{2} c_{6}-8 c_{8}\end{array}\right)$

$\operatorname{ch}_{9}=\frac{1}{9 !}\left(\begin{array}{l}-c_{1}^{9}+9 c_{2} c_{1}^{7}-9 c_{3} c_{1}^{6}-27 c_{2}^{2} c_{1}^{5}+9 c_{4} c_{1}^{5}+45 c_{2} c_{3} c_{1}^{4} \\ -9 c_{5} c_{1}^{4}+30 c_{2}^{3} c_{1}^{3}-18 c_{3}^{2} c_{1}^{3}-36 c_{2} c_{4} c_{1}^{3}+9 c_{6} c_{1}^{3} \\ -54 c_{2}^{2} c_{3} c_{1}^{2}+27 c_{3} c_{4} c_{1}^{2}+27 c_{2} c_{5} c_{1}^{2}-9 c_{9}-9 c_{7} c_{1}^{2} \\ -9 c_{2}^{4} c_{1}+27 c_{2} c_{3}^{2} c_{1}-9 c_{4}^{2} c_{1}+27 c_{2}^{2} c_{4} c_{1}-18 c_{3} c_{5} c_{1} \\ -18 c_{2} c_{6} c_{1}+9 c_{8} c_{1}-3 c_{3}^{3}+9 c_{2}^{3} c_{3} \\ -18 c_{2} c_{3} c_{4}-9 c_{2}^{2} c_{5}+9 c_{4} c_{5}+9 c_{3} c_{6}+9 c_{2} c_{7}\end{array}\right)$ 


$$
\operatorname{ch}_{10}=\frac{1}{10 !}\left(\begin{array}{l}
c_{1}^{10}-10 c_{2} c_{1}^{8}+10 c_{3} c_{1}^{7}+35 c_{2}^{2} c_{1}^{6}-10 c_{4} c_{1}^{6}-60 c_{2} c_{3} c_{1}^{5} \\
10 c_{5} c_{1}^{5}-50 c_{2}^{3} c_{1}^{4}+25 c_{3}^{2} c_{1}^{4}+50 c_{2} c_{4} c_{1}^{4}-10 c_{6} c_{1}^{4} \\
100 c_{2}^{2} c_{3} c_{1}^{3}-40 c_{3} c_{4} c_{1}^{3}-40 c_{2} c_{5} c_{1}^{3}+10 c_{7} c_{1}^{3}+25 c_{2}^{4} c_{1}^{2} \\
15 c_{4}^{2} c_{1}^{2}-60 c_{2} c_{3}^{2} c_{1}^{2}-60 c_{2}^{2} c_{4} c_{1}^{2}+30 c_{3} c_{5} c_{1}^{2}+30 c_{2} c_{6} c_{1}^{2} \\
10 c_{3}^{3} c_{1}-10 c_{8} c_{1}^{2}-40 c_{2}^{3} c_{3} c_{1}+60 c_{2} c_{3} c_{4} c_{1}+30 c_{2}^{2} c_{5} c_{1} \\
10 c_{9} c_{1}-20 c_{4} c_{5} c_{1}-20 c_{3} c_{6} c_{1}-20 c_{2} c_{7} c_{1}-2 c_{2}^{5} \\
15 c_{2}^{2} c_{3}^{2}-10 c_{2} c_{4}^{2}+5 c_{5}^{2}+10 c_{2}^{3} c_{4}-10 c_{3}^{2} c_{4}-20 c_{2} c_{3} c_{5} \\
10 c_{4} c_{6}-10 c_{2}^{2} c_{6}+10 c_{3} c_{7}+10 c_{2} c_{8}-10 c_{10}
\end{array}\right) .
$$

\section{$\operatorname{ch}\left(\Omega_{10}^{2}\right)$ :}

$$
\begin{aligned}
& \operatorname{ch}_{0}=45 ; \quad \operatorname{ch}_{1}=-9 c_{1} ; \quad \operatorname{ch}_{2}=\frac{1}{2}\left(9 c_{1}^{2}-16 c_{2}\right) ; \\
& \operatorname{ch}_{3}=\frac{1}{2}\left(-3 c_{1}^{3}+8 c_{2} c_{1}-6 c_{3}\right) \\
& \mathrm{ch}_{4}=\frac{1}{4 !}\left(9 c_{1}^{4}-32 c_{2} c_{1}^{2}+20 c_{3} c_{1}+16 c_{2}^{2}-8 c_{4}\right) \text {; } \\
& \operatorname{ch}_{5}=\frac{1}{5 !}\left(\begin{array}{l}
-9 c_{1}^{5}+40 c_{2} c_{1}^{3}-20 c_{3} c_{1}^{2}-40 c_{2}^{2} c_{1} \\
-10 c_{4} c_{1}+30 c_{2} c_{3}+30 c_{5}
\end{array}\right) \\
& \operatorname{ch}_{6}=\frac{1}{6 !}\left(\begin{array}{l}
9 c_{1}^{6}-48 c_{2} c_{1}^{4}+18 c_{3} c_{1}^{3}+72 c_{2}^{2} c_{1}^{2}+42 c_{4} c_{1}^{2}-102 c_{5} c_{1} \\
-66 c_{2} c_{3} c_{1}-16 c_{2}^{3}+24 c_{3}^{2}-12 c_{2} c_{4}+132 c_{6}
\end{array}\right) \\
& \operatorname{ch}_{7}=\frac{1}{7 !}\left(\begin{array}{l}
56 c_{2} c_{1}^{5}-9 c_{1}^{7}-14 c_{3} c_{1}^{4}-112 c_{2}^{2} c_{1}^{3}-91 c_{4} c_{1}^{3} \\
105 c_{2} c_{3} c_{1}^{2}+231 c_{5} c_{1}^{2}+56 c_{2}^{3} c_{1}-63 c_{3}^{2} c_{1}+84 c_{2} c_{4} c_{1} \\
42 c_{3} c_{4}-336 c_{6} c_{1}-42 c_{2}^{2} c_{3}-168 c_{2} c_{5}+378 c_{7}
\end{array}\right) \\
& \operatorname{ch}_{8}=\frac{1}{8 !}\left(\begin{array}{l}
9 c_{1}^{8}-64 c_{2} c_{1}^{6}+8 c_{3} c_{1}^{5}+160 c_{2}^{2} c_{1}^{4}+160 c_{4} c_{1}^{4} \\
-144 c_{2} c_{3} c_{1}^{3}-440 c_{5} c_{1}^{3}-128 c_{2}^{3} c_{1}^{2}+124 c_{3}^{2} c_{1}^{2} \\
-256 c_{2} c_{4} c_{1}^{2}+720 c_{6} c_{1}^{2}+136 c_{2}^{2} c_{3} c_{1}-128 c_{3} c_{4} c_{1} \\
+656 c_{2} c_{5} c_{1}-888 c_{7} c_{1}+16 c_{2}^{4}-64 c_{2} c_{3}^{2}+88 c_{4}^{2} \\
+48 c_{2}^{2} c_{4}-104 c_{3} c_{5}-608 c_{2} c_{6}+944 c_{8}
\end{array}\right) \\
& \operatorname{ch}_{9}=\frac{3}{9 !}\left(\begin{array}{l}
-3 c_{1}^{9}+24 c_{2} c_{1}^{7}-72 c_{2}^{2} c_{1}^{5}-84 c_{4} c_{1}^{5}+60 c_{2} c_{3} c_{1}^{4} \\
+252 c_{5} c_{1}^{4}+80 c_{2}^{3} c_{1}^{3}-72 c_{3}^{2} c_{1}^{3}+192 c_{2} c_{4} c_{1}^{3} \\
-462 c_{6} c_{1}^{3}-96 c_{2}^{2} c_{3} c_{1}^{2}+102 c_{3} c_{4} c_{1}^{2}-570 c_{2} c_{5} c_{1}^{2} \\
+630 c_{7} c_{1}^{2}-24 c_{2}^{4} c_{1}+78 c_{2} c_{3}^{2} c_{1}-114 c_{4}^{2} c_{1} \\
-90 c_{2}^{2} c_{4} c_{1}+108 c_{3} c_{5} c_{1}+780 c_{2} c_{6} c_{1}-714 c_{8} c_{1} \\
-6 c_{3}^{3}+18 c_{2}^{3} c_{3}-36 c_{2} c_{3} c_{4}+150 c_{2}^{2} c_{5}+102 c_{4} c_{5} \\
-234 c_{3} c_{6}-570 c_{2} c_{7}+738 c_{9}
\end{array}\right)
\end{aligned}
$$




$$
\operatorname{ch}_{10}=\frac{1}{10 !}\left(\begin{array}{l}
9 c_{1}^{10}-80 c_{2} c_{1}^{8}-10 c_{3} c_{1}^{7}+280 c_{2}^{2} c_{1}^{6}+370 c_{4} c_{1}^{6} \\
-210 c_{2} c_{3} c_{1}^{5}-400 c_{2}^{3} c_{1}^{4}+350 c_{3}^{2} c_{1}^{4}-1100 c_{2} c_{4} c_{1}^{4} \\
+2470 c_{6} c_{1}^{4}+500 c_{2}^{2} c_{3} c_{1}^{3}-650 c_{3} c_{4} c_{1}^{3}+3670 c_{2} c_{5} c_{1}^{3} \\
-3730 c_{7} c_{1}^{3}+200 c_{2}^{4} c_{1}^{2}-570 c_{2} c_{3}^{2} c_{1}^{2}+930 c_{4}^{2} c_{1}^{2} \\
+870 c_{2}^{2} c_{4} c_{1}^{2}-660 c_{3} c_{5} c_{1}^{2}-6240 c_{2} c_{6} c_{1}^{2}+4570 c_{8} c_{1}^{2} \\
+50 c_{3}^{3} c_{1}-230 c_{2}^{3} c_{3} c_{1}+480 c_{2} c_{3} c_{4} c_{1}-2190 c_{2}^{2} c_{5} c_{1} \\
-1390 c_{4} c_{5} c_{1}+2390 c_{3} c_{6} c_{1}+6710 c_{2} c_{7} c_{1}+20 c_{4} c_{6} \\
-4930 c_{9} c_{1}-16 c_{2}^{5}+120 c_{2}^{2} c_{3}^{2}-380 c_{2} c_{4}^{2}+640 c_{5}^{2} \\
-100 c_{2}^{3} c_{4}-20 c_{3}^{2} c_{4}+500 c_{2} c_{3} c_{5}+1780 c_{2}^{2} c_{6} \\
-2500 c_{3} c_{7}-4300 c_{2} c_{8}-1210 c_{5} c_{1}^{5}+5020 c_{10}
\end{array}\right)
$$

$$
\begin{aligned}
& \operatorname{ch}\left(\Omega_{10}^{3}\right) \text { : } \\
& \operatorname{ch}_{0}=120 ; \quad \operatorname{ch}_{1}=-36 c_{1} ; \quad \operatorname{ch}_{2}=2\left(9 c_{1}^{2}-14 c_{2}\right) ; \\
& \operatorname{ch}_{3}=-6 c_{1}^{3}+14 c_{2} c_{1}-7 c_{3} ; \\
& \mathrm{ch}_{4}=\frac{4}{4 !}\left(9 c_{1}^{4}-28 c_{2} c_{1}^{2}+7 c_{3} c_{1}+14 c_{2}^{2}+8 c_{4}\right) \text {; } \\
& \mathrm{ch}_{5}=\frac{2}{5 !}\left(-18 c_{1}^{5}+70 c_{2} c_{1}^{3}-70 c_{2}^{2} c_{1}-75 c_{4} c_{1}+35 c_{2} c_{3}+85 c_{5}\right) ; \\
& \operatorname{ch}_{6}=\frac{2}{6 !}\left(\begin{array}{l}
18 c_{1}^{6}-84 c_{2} c_{1}^{4}-21 c_{3} c_{1}^{3}+126 c_{2}^{2} c_{1}^{2}+171 c_{4} c_{1}^{2} \\
42 c_{3}^{2}-63 c_{2} c_{3} c_{1}-201 c_{5} c_{1}-28 c_{2}^{3}-96 c_{2} c_{4}+96 c_{6}
\end{array}\right) ; \\
& \operatorname{ch}_{7}=\frac{1}{7 !}\left(\begin{array}{l}
-36 c_{1}^{7}+196 c_{2} c_{1}^{5}+98 c_{3} c_{1}^{4}-392 c_{2}^{2} c_{1}^{3}-623 c_{4} c_{1}^{3} \\
+147 c_{2} c_{3} c_{1}^{2}+763 c_{5} c_{1}^{2}+196 c_{2}^{3} c_{1}-245 c_{3}^{2} c_{1} \\
+770 c_{2} c_{4} c_{1}-28 c_{6} c_{1}-98 c_{2}^{2} c_{3}+98 c_{3} c_{4} \\
-742 c_{2} c_{5}-938 c_{7}
\end{array}\right) \\
& \operatorname{ch}_{8}=\frac{4}{8 !}\left(\begin{array}{l}
9 c_{1}^{8}-56 c_{2} c_{1}^{6}-42 c_{3} c_{1}^{5}+140 c_{2}^{2} c_{1}^{4}+252 c_{4} c_{1}^{4} \\
-28 c_{2} c_{3} c_{1}^{3}-322 c_{5} c_{1}^{3}-112 c_{2}^{3} c_{1}^{2}+133 c_{3}^{2} c_{1}^{2} \\
-490 c_{2} c_{4} c_{1}^{2}-168 c_{6} c_{1}^{2}+70 c_{2}^{2} c_{3} c_{1}-98 c_{3} c_{4} c_{1} \\
+658 c_{2} c_{5} c_{1}+1134 c_{7} c_{1}+14 c_{2}^{4}-56 c_{2} c_{3}^{2}+112 c_{4}^{2} \\
+112 c_{2}^{2} c_{4}-196 c_{3} c_{5}-112 c_{2} c_{6}-1904 c_{8}
\end{array}\right) \\
& \operatorname{ch}_{9}=\frac{6}{9 !}\left(\begin{array}{l}
-6 c_{1}^{9}+42 c_{2} c_{1}^{7}+42 c_{3} c_{1}^{6}-126 c_{2}^{2} c_{1}^{5}-252 c_{4} c_{1}^{5} \\
+336 c_{5} c_{1}^{4}+140 c_{2}^{3} c_{1}^{3}-168 c_{3}^{2} c_{1}^{3}+672 c_{2} c_{4} c_{1}^{3} \\
+399 c_{6} c_{1}^{3}-84 c_{2}^{2} c_{3} c_{1}^{2}+189 c_{3} c_{4} c_{1}^{2}-1071 c_{2} c_{5} c_{1}^{2} \\
-2331 c_{7} c_{1}^{2}-42 c_{2}^{4} c_{1}+147 c_{2} c_{3}^{2} c_{1}-315 c_{4}^{2} c_{1} \\
-357 c_{2}^{2} c_{4} c_{1}+462 c_{3} c_{5} c_{1}+42 c_{2} c_{6} c_{1}+4641 c_{8} c_{1} \\
-7 c_{3}^{3}+21 c_{2}^{3} c_{3}-42 c_{2} c_{3} c_{4}+315 c_{2}^{2} c_{5}+189 c_{4} c_{5} \\
-483 c_{3} c_{6}+1365 c_{2} c_{7}-6069 c_{9}
\end{array}\right)
\end{aligned}
$$




$$
\operatorname{ch}_{10}=\frac{2}{10 !}\left(\begin{array}{l}
18 c_{1}^{10}-140 c_{2} c_{1}^{8}-175 c_{3} c_{1}^{7}+490 c_{2}^{2} c_{1}^{6}+1075 c_{4} c_{1}^{6} \\
105 c_{2} c_{3} c_{1}^{5}-1495 c_{5} c_{1}^{5}-700 c_{2}^{3} c_{1}^{4}+875 c_{3}^{2} c_{1}^{4} \\
350 c_{2}^{2} c_{3} c_{1}^{3}-3650 c_{2} c_{4} c_{1}^{4}-2915 c_{6} c_{1}^{4}-1355 c_{3} c_{4} c_{1}^{3} \\
6565 c_{2} c_{5} c_{1}^{3}+17405 c_{7} c_{1}^{3}+350 c_{2}^{4} c_{1}^{2}-1155 c_{2} c_{3}^{2} c_{1}^{2} \\
2685 c_{4}^{2} c_{1}^{2}+3165 c_{2}^{2} c_{4} c_{1}^{2}-3450 c_{3} c_{5} c_{1}^{2} \\
1230 c_{2} c_{6} c_{1}^{2}-40505 c_{8} c_{1}^{2}+35 c_{3}^{3} c_{1}-245 c_{2}^{3} c_{3} c_{1} \\
750 c_{2} c_{3} c_{4} c_{1}-4395 c_{2}^{2} c_{5} c_{1}-2665 c_{4} c_{5} c_{1} \\
6155 c_{3} c_{6} c_{1}-21205 c_{2} c_{7} c_{1}+61925 c_{9} c_{1}-28 c_{2}^{5} \\
210 c_{2}^{2} c_{3}^{2}-1040 c_{2} c_{4}^{2}+1870 c_{5}^{2}-400 c_{2}^{3} c_{4} \\
40 c_{3}^{2} c_{4}+1700 c_{2} c_{3} c_{5}+400 c_{2}^{2} c_{6}-2560 c_{4} c_{6} \\
29840 c_{2} c_{8}-40 c_{3} c_{7}-73040 c_{10}
\end{array}\right)
$$

$\operatorname{ch}\left(\Omega_{10}^{4}\right)$

$\operatorname{ch}_{0}=210 ; \quad \operatorname{ch}_{1}=-84 c_{1} ; \quad \operatorname{ch}_{2}=14\left(3 c_{1}^{2}-4 c_{2}\right) ;$

$\operatorname{ch}_{3}=-7\left(2 c_{1}^{3}-4 c_{2} c_{1}+c_{3}\right)$;

$\mathrm{ch}_{4}=\frac{4}{4 !}\left(21 c_{1}^{4}-56 c_{2} c_{1}^{2}-7 c_{3} c_{1}+28 c_{2}^{2}+34 c_{4}\right)$;

$\operatorname{ch}_{5}=\frac{2}{5 !}\left(\begin{array}{l}-42 c_{1}^{5}+140 c_{2} c_{1}^{3}+70 c_{3} c_{1}^{2}-140 c_{2}^{2} c_{1} \\ -205 c_{4} c_{1}+35 c_{2} c_{3}+115 c_{5}\end{array}\right)$

$\operatorname{ch}_{6}=\frac{2}{6 !}\left(\begin{array}{l}42 c_{1}^{6}-168 c_{2} c_{1}^{4}-147 c_{3} c_{1}^{3}+252 c_{2}^{2} c_{1}^{2}+417 c_{4} c_{1}^{2} \\ 84 c_{3}^{2}-21 c_{2} c_{3} c_{1}-147 c_{5} c_{1}-56 c_{2}^{3}-282 c_{2} c_{4}-258 c_{6}\end{array}\right) ;$

$\operatorname{ch}_{7}=\frac{7}{7 !}\left(\begin{array}{l}-12 c_{1}^{7}+56 c_{2} c_{1}^{5}+70 c_{3} c_{1}^{4}-112 c_{2}^{2} c_{1}^{3}-205 c_{4} c_{1}^{3} \\ -21 c_{2} c_{3} c_{1}^{2}+25 c_{5} c_{1}^{2}+56 c_{2}^{3} c_{1}-77 c_{3}^{2} c_{1}+296 c_{2} c_{4} c_{1} \\ +380 c_{6} c_{1}-14 c_{2}^{2} c_{3}+14 c_{3} c_{4}-136 c_{2} c_{5}-434 c_{7}\end{array}\right)$

$\operatorname{ch}_{8}=\frac{28}{8 !}\left(\begin{array}{l}3 c_{1}^{8}-16 c_{2} c_{1}^{6}-26 c_{3} c_{1}^{5}+40 c_{2}^{2} c_{1}^{4}+80 c_{4} c_{1}^{4}+20 c_{2} c_{3} c_{1}^{3} \\ +10 c_{5} c_{1}^{3}-32 c_{2}^{3} c_{1}^{2}+45 c_{3}^{2} c_{1}^{2}-180 c_{2} c_{4} c_{1}^{2}-280 c_{6} c_{1}^{2} \\ +6 c_{2}^{2} c_{3} c_{1}-20 c_{3} c_{4} c_{1}+100 c_{2} c_{5} c_{1}+334 c_{7} c_{1}+4 c_{2}^{4} \\ -16 c_{2} c_{3}^{2}+38 c_{4}^{2}+44 c_{2}^{2} c_{4}-74 c_{3} c_{5}+136 c_{2} c_{6}+44 c_{8}\end{array}\right)$

$\operatorname{ch}_{9}=\frac{42}{9 !}\left(\begin{array}{l}-2 c_{1}^{9}+12 c_{2} c_{1}^{7}+24 c_{3} c_{1}^{6}-36 c_{2}^{2} c_{1}^{5}-78 c_{4} c_{1}^{5}-30 c_{2} c_{3} c_{1}^{4} \\ -30 c_{5} c_{1}^{4}+40 c_{2}^{3} c_{1}^{3}-60 c_{3}^{2} c_{1}^{3}+240 c_{2} c_{4} c_{1}^{3}+435 c_{6} c_{1}^{3} \\ +45 c_{3} c_{4} c_{1}^{2}-135 c_{2} c_{5} c_{1}^{2}-543 c_{7} c_{1}^{2}-12 c_{2}^{4} c_{1}+45 c_{2} c_{3}^{2} c_{1} \\ -111 c_{4}^{2} c_{1}-135 c_{2}^{2} c_{4} c_{1}+198 c_{3} c_{5} c_{1}-462 c_{2} c_{6} c_{1} \\ -591 c_{8} c_{1}-c_{3}^{3}+3 c_{2}^{3} c_{3}-6 c_{2} c_{3} c_{4}+57 c_{2}^{2} c_{5} \\ +33 c_{4} c_{5}-87 c_{3} c_{6}+513 c_{2} c_{7}+2427 c_{9}\end{array}\right)$ 


$$
\operatorname{ch}_{10}=\frac{2}{10 !}\left(\begin{array}{l}
42 c_{1}^{10}-280 c_{2} c_{1}^{8}-665 c_{3} c_{1}^{7}+980 c_{2}^{2} c_{1}^{6}+2285 c_{4} c_{1}^{6} \\
+1155 c_{2} c_{3} c_{1}^{5}+1495 c_{5} c_{1}^{5}-1400 c_{2}^{3} c_{1}^{4}+2275 c_{3}^{2} c_{1}^{4} \\
-8950 c_{2} c_{4} c_{1}^{4}+420 c_{2}^{2} c_{3}^{2}-350 c_{2}^{2} c_{3} c_{1}^{3}-2425 c_{3} c_{4} c_{1}^{3} \\
+4775 c_{2} c_{5} c_{1}^{3}+24175 c_{7} c_{1}^{3}+700 c_{2}^{4} c_{1}^{2}-2625 c_{2} c_{3}^{2} c_{1}^{2} \\
+6765 c_{4}^{2} c_{1}^{2}+8175 c_{2}^{2} c_{4} c_{1}^{2}-11670 c_{3} c_{5} c_{1}^{2} \\
+55205 c_{8} c_{1}^{2}-35 c_{3}^{3} c_{1}-175 c_{2}^{3} c_{3} c_{1}+30900 c_{2} c_{6} c_{1}^{2} \\
-5055 c_{2}^{2} c_{5} c_{1}-3215 c_{4} c_{5} c_{1}+10015 c_{3} c_{6} c_{1} \\
-48305 c_{2} c_{7} c_{1}-247985 c_{9} c_{1}-18505 c_{6} c_{1}^{4} \\
-2530 c_{2} c_{4}^{2}+4640 c_{5}^{2}-1070 c_{2}^{3} c_{4}+170 c_{3}^{2} c_{4} \\
+4390 c_{2} c_{3} c_{5}-56 c_{2}^{5}-10250 c_{4} c_{6}+1140 c_{2} c_{3} c_{4} c_{1} \\
+11170 c_{3} c_{7}-6490 c_{2}^{2} c_{6}-12410 c_{2} c_{8}+441170 c_{10}
\end{array}\right)
$$

\section{$\operatorname{ch}\left(\Omega_{10}^{5}\right):$}

$$
\begin{aligned}
& \operatorname{ch}_{0}=252 ; \quad \operatorname{ch}_{1}=-126 c_{1} ; \quad \operatorname{ch}_{2}=7\left(9 c_{1}^{2}-10 c_{2}\right) ; \\
& \operatorname{ch}_{3}=-7\left(3 c_{1}^{3}-5 c_{1} c_{2}\right) ; \\
& \operatorname{ch}_{4}=\frac{2}{4 !}\left(\begin{array}{ll}
\left.6 c_{1}^{4}-140 c_{2} c_{1}^{2}-70 c_{3} c_{1}+70 c_{2}^{2}+100 c_{4}\right) ; \\
\operatorname{ch}_{5}=\frac{2}{5 !}\left(-63 c_{1}^{5}+175 c_{2} c_{1}^{3}+175 c_{3} c_{1}^{2}-175 c_{2}^{2} c_{1}-250 c_{4} c_{1}\right) ; \\
\operatorname{ch}_{6}=\frac{2}{6 !}\left(\begin{array}{l}
63 c_{1}^{6}-210 c_{2} c_{1}^{4}-315 c_{3} c_{1}^{3}+315 c_{2}^{2} c_{1}^{2}+465 c_{4} c_{1}^{2}-70 c_{2}^{3} \\
+105 c_{2} c_{3} c_{1}+285 c_{5} c_{1}+105 c_{3}^{2}-390 c_{2} c_{4}-570 c_{6}
\end{array}\right) ; \\
\operatorname{ch}_{7}=\frac{7}{7 !}\left(\begin{array}{l}
70 c_{2} c_{1}^{5}-18 c_{1}^{7}+140 c_{3} c_{1}^{4}-140 c_{2}^{2} c_{1}^{3}-215 c_{4} c_{1}^{3}+70 c_{2}^{3} c_{1} \\
390 c_{2} c_{4} c_{1}-285 c_{5} c_{1}^{2}-105 c_{3}^{2} c_{1}+570 c_{6} c_{1}-105 c_{2} c_{3} c_{1}^{2}
\end{array}\right) ; \\
\operatorname{ch}_{8}=\frac{14}{8 !}\left(\begin{array}{l}
9 c_{1}^{8}-40 c_{2} c_{1}^{6}-100 c_{3} c_{1}^{5}+100 c_{2}^{2} c_{1}^{4}+160 c_{4} c_{1}^{4} \\
+120 c_{2} c_{3} c_{1}^{3}+340 c_{5} c_{1}^{3}-80 c_{2}^{3} c_{1}^{2}+130 c_{3}^{2} c_{1}^{2} \\
-460 c_{2} c_{4} c_{1}^{2}-720 c_{6} c_{1}^{2}-20 c_{2}^{2} c_{3} c_{1}-20 c_{3} c_{4} c_{1} \\
-100 c_{2} c_{5} c_{1}-420 c_{7} c_{1}+10 c_{2}^{4}-40 c_{2} c_{3}^{2}+100 c_{4}^{2} \\
+120 c_{2}^{2} c_{4}-200 c_{3} c_{5}+520 c_{2} c_{6}+1400 c_{8}
\end{array}\right) ; \\
\operatorname{ch}_{9}=\frac{42}{9 !}\left(\begin{array}{l}
15 c_{2} c_{1}^{7}-3 c_{1}^{9}+45 c_{3} c_{1}^{6}-45 c_{2}^{2} c_{1}^{5}-75 c_{4} c_{1}^{5} \\
50 c_{2}^{3} c_{1}^{3}-75 c_{2} c_{3} c_{1}^{4}-225 c_{5} c_{1}^{4}-90 c_{3}^{2} c_{1}^{3}+300 c_{2} c_{4} c_{1}^{3} \\
510 c_{6} c_{1}^{3}+30 c_{2}^{2} c_{3} c_{1}^{2}+30 c_{3} c_{4} c_{1}^{2} \\
150 c_{2} c_{5} c_{1}^{2}+630 c_{7} c_{1}^{2}-15 c_{2}^{4} c_{1}+60 c_{2} c_{3}^{2} c_{1}-150 c_{4}^{2} c_{1} \\
300 c_{3} c_{5} c_{1}-180 c_{2}^{2} c_{4} c_{1}-780 c_{2} c_{6} c_{1}-2100 c_{8} c_{1}
\end{array}\right) ;
\end{array}\right) ;
\end{aligned}
$$




$$
\operatorname{ch}_{10}=\frac{2}{10 !}\left(\begin{array}{l}
63 c_{1}^{10}-350 c_{2} c_{1}^{8}-1225 c_{3} c_{1}^{7}+1225 c_{2}^{2} c_{1}^{6}+2125 c_{4} c_{1}^{6} \\
+2625 c_{2} c_{3} c_{1}^{5}+8375 c_{5} c_{1}^{5}-1750 c_{2}^{3} c_{1}^{4}+3500 c_{3}^{2} c_{1}^{4} \\
-11000 c_{2} c_{4} c_{1}^{4}-20345 c_{6} c_{1}^{4}-1750 c_{2}^{2} c_{3} c_{1}^{3} \\
-1775 c_{3} c_{4} c_{1}^{3}-8975 c_{2} c_{5} c_{1}^{3}-39505 c_{7} c_{1}^{3}+875 c_{2}^{4} c_{1}^{2} \\
-3675 c_{2} c_{3}^{2} c_{1}^{2}+9300 c_{4}^{2} c_{1}^{2}+10725 c_{2}^{2} c_{4} c_{1}^{2} \\
-19200 c_{3} c_{5} c_{1}^{2}+48480 c_{2} c_{6} c_{1}^{2}+142405 c_{8} c_{1}^{2} \\
-175 c_{3}^{3} c_{1}+175 c_{2}^{3} c_{3} c_{1}+750 c_{2} c_{3} c_{4} c_{1} \\
+1275 c_{2}^{2} c_{5} c_{1}+275 c_{4} c_{5} c_{1}+2795 c_{3} c_{6} c_{1} \\
+10715 c_{2} c_{7} c_{1}+78095 c_{9} c_{1}-70 c_{2}^{5}+525 c_{2}^{2} c_{3}^{2} \\
-3350 c_{2} c_{4}^{2}+6175 c_{5}^{2}-1450 c_{2}^{3} c_{4}+250 c_{3}^{2} c_{4} \\
+5900 c_{2} c_{3} c_{5}-11990 c_{2}^{2} c_{6}-15370 c_{4} c_{6} \\
+19910 c_{3} c_{7}-88810 c_{2} c_{8}-780950 c_{10}
\end{array}\right) .
$$

$\operatorname{ch}\left(\Omega_{10}^{6}\right):$

$$
\begin{aligned}
& \operatorname{ch}_{0}=210 ; \quad \operatorname{ch}_{1}=-126 c_{1} ; \quad \operatorname{ch}_{2}=7\left(9 c_{1}^{2}-8 c_{2}\right) ; \\
& \operatorname{ch}_{3}=-7\left(3 c_{1}^{3}-4 c_{2} c_{1}-c_{3}\right) \text {; } \\
& \mathrm{ch}_{4}=\frac{2}{4 !}\left(63 c_{1}^{4}-112 c_{2} c_{1}^{2}-98 c_{3} c_{1}+56 c_{2}^{2}+68 c_{4}\right) ; \\
& \operatorname{ch}_{5}=\frac{2}{5 !}\left(\begin{array}{l}
-63 c_{1}^{5}+140 c_{2} c_{1}^{3}+210 c_{3} c_{1}^{2}-140 c_{2}^{2} c_{1} \\
-135 c_{4} c_{1}-35 c_{2} c_{3}-115 c_{5}
\end{array}\right) \\
& \operatorname{ch}_{6}=\frac{2}{6 !}\left(\begin{array}{l}
63 c_{1}^{6}-168 c_{2} c_{1}^{4}-357 c_{3} c_{1}^{3}+252 c_{2}^{2} c_{1}^{2}+207 c_{4} c_{1}^{2}-56 c_{2}^{3} \\
+189 c_{2} c_{3} c_{1}+543 c_{5} c_{1}+84 c_{3}^{2}-282 c_{2} c_{4}-258 c_{6}
\end{array}\right) ; \\
& \operatorname{ch}_{7}=\frac{7}{7 !}\left(\begin{array}{l}
-18 c_{1}^{7}+56 c_{2} c_{1}^{5}+154 c_{3} c_{1}^{4}-112 c_{2}^{2} c_{1}^{3}-79 c_{4} c_{1}^{3} \\
-421 c_{5} c_{1}^{2}+56 c_{2}^{3} c_{1}-91 c_{3}^{2} c_{1}+268 c_{2} c_{4} c_{1}-147 c_{2} c_{3} c_{1}^{2} \\
+136 c_{6} c_{1}+14 c_{2}^{2} c_{3}-14 c_{3} c_{4}+136 c_{2} c_{5}+434 c_{7}
\end{array}\right) ; \\
& \operatorname{ch}_{8}=\frac{14}{8 !}\left(\begin{array}{l}
9 c_{1}^{8}-32 c_{2} c_{1}^{6}-108 c_{3} c_{1}^{5}+80 c_{2}^{2} c_{1}^{4}+48 c_{4} c_{1}^{4}-72 c_{6} c_{1}^{2} \\
452 c_{5} c_{1}^{3}-64 c_{2}^{3} c_{1}^{2}+118 c_{3}^{2} c_{1}^{2}-304 c_{2} c_{4} c_{1}^{2}+152 c_{2} c_{3} c_{1}^{3} \\
16 c_{3} c_{4} c_{1}-44 c_{2}^{2} c_{3} c_{1}-344 c_{2} c_{5} c_{1}-1068 c_{7} c_{1}+8 c_{2}^{4} \\
76 c_{4}^{2}-32 c_{2} c_{3}^{2}+88 c_{2}^{2} c_{4}-148 c_{3} c_{5}+272 c_{2} c_{6}+88 c_{8}
\end{array}\right) \\
& \operatorname{ch}_{9}=\frac{42}{9 !}\left(\begin{array}{l}
-3 c_{1}^{9}+12 c_{2} c_{1}^{7}+48 c_{3} c_{1}^{6}-36 c_{2}^{2} c_{1}^{5}-18 c_{4} c_{1}^{5} \\
-282 c_{5} c_{1}^{4}+40 c_{2}^{3} c_{1}^{3}-84 c_{3}^{2} c_{1}^{3}+192 c_{2} c_{4} c_{1}^{3}-3 c_{6} c_{1}^{3} \\
+48 c_{2}^{2} c_{3} c_{1}^{2}-9 c_{3} c_{4} c_{1}^{2}+351 c_{2} c_{5} c_{1}^{2}+1143 c_{7} c_{1}^{2} \\
-12 c_{2}^{4} c_{1}+51 c_{2} c_{3}^{2} c_{1}-117 c_{4}^{2} c_{1}-129 c_{2}^{2} c_{4} c_{1} \\
+246 c_{3} c_{5} c_{1}-354 c_{2} c_{6} c_{1}+327 c_{8} c_{1}+c_{3}^{3}-3 c_{2}^{3} c_{3} \\
+6 c_{2} c_{3} c_{4}-57 c_{2}^{2} c_{5}-33 c_{4} c_{5}-90 c_{2} c_{3} c_{1}^{4} \\
+87 c_{3} c_{6}-513 c_{2} c_{7}-2427 c_{9}
\end{array}\right)
\end{aligned}
$$




$$
\operatorname{ch}_{10}=\frac{2}{10 !}\left(\begin{array}{l}
63 c_{1}^{10}-280 c_{2} c_{1}^{8}-1295 c_{3} c_{1}^{7}+980 c_{2}^{2} c_{1}^{6}+395 c_{4} c_{1}^{6} \\
+3045 c_{2} c_{3} c_{1}^{5}+10105 c_{5} c_{1}^{5}-1400 c_{2}^{3} c_{1}^{4}+3325 c_{3}^{2} c_{1}^{4} \\
-6850 c_{2} c_{4} c_{1}^{4}+1865 c_{6} c_{1}^{4}-2450 c_{2}^{2} c_{3} c_{1}^{3}+305 c_{3} c_{4} c_{1}^{3} \\
-17695 c_{2} c_{5} c_{1}^{3}-61715 c_{7} c_{1}^{3}+700 c_{2}^{4} c_{1}^{2}-3255 c_{2} c_{3}^{2} c_{1}^{2} \\
+7395 c_{4}^{2} c_{1}^{2}+7545 c_{2}^{2} c_{4} c_{1}^{2}-16710 c_{3} c_{5} c_{1}^{2} \\
+19560 c_{2} c_{6} c_{1}^{2}-41185 c_{8} c_{1}^{2}-245 c_{3}^{3} c_{1}+455 c_{2}^{3} c_{3} c_{1} \\
-120 c_{2} c_{3} c_{4} c_{1}+6915 c_{2}^{2} c_{5} c_{1}+3715 c_{4} c_{5} c_{1} \\
-8255 c_{3} c_{6} c_{1}+59425 c_{2} c_{7} c_{1}+261685 c_{9} c_{1}-56 c_{2}^{5} \\
+420 c_{2}^{2} c_{3}^{2}-2530 c_{2} c_{4}^{2}+4640 c_{5}^{2}-1070 c_{2}^{3} c_{4} \\
+4390 c_{2} c_{3} c_{5}-6490 c_{2}^{2} c_{6}-10250 c_{4} c_{6}+170 c_{3}^{2} c_{4} \\
+11170 c_{3} c_{7}-12410 c_{2} c_{8}+441170 c_{10}
\end{array}\right)
$$

\section{$\operatorname{ch}\left(\Omega_{10}^{7}\right)$ :}

$$
\begin{aligned}
& \operatorname{ch}_{0}=120 ; \quad \operatorname{ch}_{1}=-84 c_{1} ; \quad \operatorname{ch}_{2}=14\left(3 c_{1}^{2}-2 c_{2}\right) ; \\
& \operatorname{ch}_{3}=\frac{1}{3 !}\left(-84 c_{1}^{3}+84 c_{2} c_{1}+42 c_{3}\right) \\
& \mathrm{ch}_{4}=\frac{4}{4 !}\left(21 c_{1}^{4}-28 c_{2} c_{1}^{2}-35 c_{3} c_{1}+14 c_{2}^{2}+8 c_{4}\right) \text {; } \\
& \operatorname{ch}_{5}=\frac{2}{5 !}\left(\begin{array}{l}
-42 c_{1}^{5}+70 c_{2} c_{1}^{3}+140 c_{3} c_{1}^{2}-70 c_{2}^{2} c_{1} \\
-5 c_{4} c_{1}-35 c_{2} c_{3}-85 c_{5}
\end{array}\right) \\
& \operatorname{ch}_{6}=\frac{2}{6 !}\left(\begin{array}{l}
42 c_{1}^{6}-84 c_{2} c_{1}^{4}-231 c_{3} c_{1}^{3}+126 c_{2}^{2} c_{1}^{2}-39 c_{4} c_{1}^{2} \\
+147 c_{2} c_{3} c_{1}+309 c_{5} c_{1}-28 c_{2}^{3}+42 c_{3}^{2}-96 c_{2} c_{4}+96 c_{6}
\end{array}\right) \\
& \operatorname{ch}_{7}=\frac{7}{7 !}\left(\begin{array}{l}
-12 c_{1}^{7}+28 c_{2} c_{1}^{5}+98 c_{3} c_{1}^{4}-56 c_{2}^{2} c_{1}^{3}+37 c_{4} c_{1}^{3} \\
-217 c_{5} c_{1}^{2}+28 c_{2}^{3} c_{1}-49 c_{3}^{2} c_{1}+82 c_{2} c_{4} c_{1}-188 c_{6} c_{1} \\
+14 c_{2}^{2} c_{3}-14 c_{3} c_{4}+106 c_{2} c_{5}-105 c_{2} c_{3} c_{1}^{2}+134 c_{7}
\end{array}\right) \\
& \operatorname{ch}_{8}=\frac{28}{8 !}\left(\begin{array}{c}
3 c_{1}^{8}-8 c_{2} c_{1}^{6}-34 c_{3} c_{1}^{5}+20 c_{2}^{2} c_{1}^{4}-20 c_{4} c_{1}^{4}+52 c_{2} c_{3} c_{1}^{3} \\
+110 c_{5} c_{1}^{3}-16 c_{2}^{3} c_{1}^{2}+33 c_{3}^{2} c_{1}^{2}-42 c_{2} c_{4} c_{1}^{2}+160 c_{6} c_{1}^{2} \\
-18 c_{2}^{2} c_{3} c_{1}+14 c_{3} c_{4} c_{1}-118 c_{2} c_{5} c_{1}-106 c_{7} c_{1}+2 c_{2}^{4} \\
-8 c_{2} c_{3}^{2}+16 c_{4}^{2}+16 c_{2}^{2} c_{4}-28 c_{3} c_{5}-16 c_{2} c_{6}-272 c_{8}
\end{array}\right) \\
& \operatorname{ch}_{9}=\frac{42}{9 !}\left(\begin{array}{l}
-2 c_{1}^{9}+6 c_{2} c_{1}^{7}+30 c_{3} c_{1}^{6}-18 c_{2}^{2} c_{1}^{5}+24 c_{4} c_{1}^{5}-60 c_{2} c_{3} c_{1}^{4} \\
-132 c_{5} c_{1}^{4}+20 c_{2}^{3} c_{1}^{3}-48 c_{3}^{2} c_{1}^{3}+48 c_{2} c_{4} c_{1}^{3}-273 c_{6} c_{1}^{3} \\
+36 c_{2}^{2} c_{3} c_{1}^{2}-27 c_{3} c_{4} c_{1}^{2}+225 c_{2} c_{5} c_{1}^{2}+165 c_{7} c_{1}^{2}-6 c_{2}^{4} c_{1} \\
+27 c_{2} c_{3}^{2} c_{1}-51 c_{4}^{2} c_{1}-45 c_{2}^{2} c_{4} c_{1}+102 c_{3} c_{5} c_{1} \\
+90 c_{2} c_{6} c_{1}+969 c_{8} c_{1}+c_{3}^{3}-3 c_{2}^{3} c_{3}+6 c_{2} c_{3} c_{4} \\
-45 c_{2}^{2} c_{5}-27 c_{4} c_{5}+69 c_{3} c_{6}-195 c_{2} c_{7}+867 c_{9}
\end{array}\right) ;
\end{aligned}
$$




$$
\operatorname{ch}_{10}=\frac{2}{10 !}\left(\begin{array}{l}
42 c_{1}^{10}-140 c_{2} c_{1}^{8}-805 c_{3} c_{1}^{7}+490 c_{2}^{2} c_{1}^{6}-815 c_{4} c_{1}^{6} \\
+1995 c_{2} c_{3} c_{1}^{5}+4595 c_{5} c_{1}^{5}-700 c_{2}^{3} c_{1}^{4}+1925 c_{3}^{2} c_{1}^{4} \\
-1550 c_{2} c_{4} c_{1}^{4}+12415 c_{6} c_{1}^{4}-1750 c_{2}^{2} c_{3} c_{1}^{3} \\
+1375 c_{3} c_{4} c_{1}^{3}-10865 c_{2} c_{5} c_{1}^{3}-6745 c_{7} c_{1}^{3}+350 c_{2}^{4} c_{1}^{2} \\
-1785 c_{2} c_{3}^{2} c_{1}^{2}+3315 c_{4}^{2} c_{1}^{2}+2535 c_{2}^{2} c_{4} c_{1}^{2} \\
-7590 c_{2} c_{6} c_{1}^{2}-72635 c_{8} c_{1}^{2}-175 c_{3}^{3} c_{1}+385 c_{2}^{3} c_{3} c_{1} \\
-510 c_{2} c_{3} c_{4} c_{1}+5055 c_{2}^{2} c_{5} c_{1}+3005 c_{4} c_{5} c_{1} \\
-8335 c_{3} c_{6} c_{1}+19745 c_{2} c_{7} c_{1}-120145 c_{9} c_{1}-28 c_{2}^{5} \\
+210 c_{2}^{2} c_{3}^{2}-1040 c_{2} c_{4}^{2}+1870 c_{5}^{2}-400 c_{2}^{3} c_{4}+40 c_{3}^{2} c_{4} \\
+1700 c_{2} c_{3} c_{5}+400 c_{2}^{2} c_{6}-7230 c_{3} c_{5} c_{1}^{2} \\
-2560 c_{4} c_{6}-40 c_{3} c_{7}+29840 c_{2} c_{8}-73040 c_{10}
\end{array}\right) .
$$

$$
\begin{aligned}
& \operatorname{ch}\left(\Omega_{10}^{8}\right) \text { : } \\
& \operatorname{ch}_{0}=45 ; \quad \operatorname{ch}_{1}=-36 c_{1} ; \quad \operatorname{ch}_{2}=\frac{1}{2}\left(36 c_{1}^{2}-16 c_{2}\right) ; \\
& \operatorname{ch}_{3}=\frac{1}{3 !}\left(-36 c_{1}^{3}+24 c_{2} c_{1}+18 c_{3}\right) \text {; } \\
& \mathrm{ch}_{4}=\frac{4}{4 !}\left(9 c_{1}^{4}-8 c_{2} c_{1}^{2}-13 c_{3} c_{1}+4 c_{2}^{2}-2 c_{4}\right) ; \\
& \operatorname{ch}_{5}=\frac{2}{5 !}\left(\begin{array}{l}
-18 c_{1}^{5}+20 c_{2} c_{1}^{3}+50 c_{3} c_{1}^{2}-20 c_{2}^{2} c_{1} \\
+25 c_{4} c_{1}-15 c_{2} c_{3}-15 c_{5}
\end{array}\right) \\
& \operatorname{ch}_{6}=\frac{2}{6 !}\left(\begin{array}{l}
18 c_{1}^{6}-24 c_{2} c_{1}^{4}-81 c_{3} c_{1}^{3}+36 c_{2}^{2} c_{1}^{2}-69 c_{4} c_{1}^{2} \\
+57 c_{2} c_{3} c_{1}+39 c_{5} c_{1}-8 c_{2}^{3}+12 c_{3}^{2}-6 c_{2} c_{4}+66 c_{6}
\end{array}\right) ; \\
& \operatorname{ch}_{7}=\frac{1}{7 !}\left(\begin{array}{l}
-36 c_{1}^{7}+56 c_{2} c_{1}^{5}+238 c_{3} c_{1}^{4}-112 c_{2}^{2} c_{1}^{3}+287 c_{4} c_{1}^{3} \\
-147 c_{5} c_{1}^{2}+56 c_{2}^{3} c_{1}-105 c_{3}^{2} c_{1}-588 c_{6} c_{1}+42 c_{2}^{2} c_{3} \\
-42 c_{3} c_{4}+168 c_{2} c_{5}-273 c_{2} c_{3} c_{1}^{2}-378 c_{7}
\end{array}\right) \\
& \operatorname{ch}_{8}=\frac{4}{8 !}\left(\begin{array}{l}
9 c_{1}^{8}-16 c_{2} c_{1}^{6}-82 c_{3} c_{1}^{5}+40 c_{2}^{2} c_{1}^{4}-128 c_{4} c_{1}^{4}+52 c_{3} c_{4} c_{1} \\
73 c_{3}^{2} c_{1}^{2}-32 c_{2}^{3} c_{1}^{2}+20 c_{2} c_{4} c_{1}^{2}+432 c_{6} c_{1}^{2}-50 c_{2}^{2} c_{3} c_{1} \\
534 c_{7} c_{1}-172 c_{2} c_{5} c_{1}+4 c_{2}^{4}-16 c_{2} c_{3}^{2}+22 c_{4}^{2}+58 c_{5} c_{1}^{3} \\
12 c_{2}^{2} c_{4}-26 c_{3} c_{5}-152 c_{2} c_{6}+132 c_{2} c_{3} c_{1}^{3}+236 c_{8}
\end{array}\right) \\
& \operatorname{ch}_{9}=\frac{6}{9 !}\left(\begin{array}{l}
-6 c_{1}^{9}+12 c_{2} c_{1}^{7}+72 c_{3} c_{1}^{6}-36 c_{2}^{2} c_{1}^{5}+138 c_{4} c_{1}^{5} \\
-150 c_{2} c_{3} c_{1}^{4}-54 c_{5} c_{1}^{4}+40 c_{2}^{3} c_{1}^{3}-108 c_{3}^{2} c_{1}^{3} \\
-48 c_{2} c_{4} c_{1}^{3}-681 c_{6} c_{1}^{3}+96 c_{2}^{2} c_{3} c_{1}^{2}-111 c_{3} c_{4} c_{1}^{2} \\
+309 c_{2} c_{5} c_{1}^{2}-1251 c_{7} c_{1}^{2}-12 c_{2}^{4} c_{1}+57 c_{2} c_{3}^{2} c_{1}-75 c_{4}^{2} c_{1} \\
-27 c_{2}^{2} c_{4} c_{1}+102 c_{3} c_{5} c_{1}+522 c_{2} c_{6} c_{1}-1059 c_{8} c_{1}+3 c_{3}^{3} \\
-9 c_{2}^{3} c_{3}+18 c_{2} c_{3} c_{4}-75 c_{2}^{2} c_{5} \\
-51 c_{4} c_{5}+117 c_{3} c_{6}+285 c_{2} c_{7}-369 c_{9}
\end{array}\right) ;
\end{aligned}
$$




$$
\operatorname{ch}_{10}=\frac{2}{10 !}\left(\begin{array}{l}
18 c_{1}^{10}-40 c_{2} c_{1}^{8}-275 c_{3} c_{1}^{7}+140 c_{2}^{2} c_{1}^{6}-625 c_{4} c_{1}^{6} \\
+705 c_{2} c_{3} c_{1}^{5}+205 c_{5} c_{1}^{5}-200 c_{2}^{3} c_{1}^{4}+625 c_{3}^{2} c_{1}^{4} \\
+350 c_{2} c_{4} c_{1}^{4}+4205 c_{6} c_{1}^{4}-650 c_{2}^{2} c_{3} c_{1}^{3}+845 c_{3} c_{4} c_{1}^{3} \\
-2035 c_{2} c_{5} c_{1}^{3}+10285 c_{7} c_{1}^{3}+100 c_{2}^{4} c_{1}^{2}-555 c_{2} c_{3}^{2} c_{1}^{2} \\
+735 c_{4}^{2} c_{1}^{2}+165 c_{2}^{2} c_{4} c_{1}^{2}-1050 c_{3} c_{5} c_{1}^{2}-5100 c_{2} c_{6} c_{1}^{2} \\
+12815 c_{8} c_{1}^{2}-65 c_{3}^{3} c_{1}+155 c_{2}^{3} c_{3} c_{1}-300 c_{2} c_{3} c_{4} c_{1} \\
+1155 c_{2}^{2} c_{5} c_{1}+835 c_{4} c_{5} c_{1}-2315 c_{3} c_{6} c_{1}+10 c_{4} c_{6} \\
-5195 c_{2} c_{7} c_{1}+8605 c_{9} c_{1}-8 c_{2}^{5}+60 c_{2}^{2} c_{3}^{2} \\
-190 c_{2} c_{4}^{2}+320 c_{5}^{2}-50 c_{2}^{3} c_{4}-10 c_{3}^{2} c_{4}+250 c_{2} c_{3} c_{5} \\
+890 c_{2}^{2} c_{6}-1250 c_{3} c_{7}-2150 c_{2} c_{8}+2510 c_{10}
\end{array}\right) .
$$

$$
\begin{aligned}
& \operatorname{ch}\left(\Omega_{10}^{9}\right) \text { : } \\
& \operatorname{ch}_{0}=10 ; \quad \operatorname{ch}_{1}=-9 c_{1} ; \quad \operatorname{ch}_{2}=\frac{1}{2}\left(9 c_{1}^{2}-2 c_{2}\right) ; \\
& \operatorname{ch}_{3}=\frac{1}{2}\left(-3 c_{1}^{3}+c_{2} c_{1}+c_{3}\right) \\
& \mathrm{ch}_{4}=\frac{1}{4 !}\left(9 c_{1}^{4}-4 c_{2} c_{1}^{2}-8 c_{3} c_{1}+2 c_{2}^{2}-4 c_{4}\right) \\
& \operatorname{ch}_{5}=\frac{1}{5 !}\left(-9 c_{1}^{5}+5 c_{2} c_{1}^{3}+15 c_{3} c_{1}^{2}-5 c_{2}^{2} c_{1}+15 c_{4} c_{1}-5 c_{2} c_{3}+5 c_{5}\right) ; \\
& \operatorname{ch}_{6}=\frac{1}{6 !}\left(\begin{array}{l}
9 c_{1}^{6}-6 c_{2} c_{1}^{4}-24 c_{3} c_{1}^{3}+9 c_{2}^{2} c_{1}^{2}-36 c_{4} c_{1}^{2}+18 c_{2} c_{3} c_{1} \\
-24 c_{5} c_{1}-2 c_{2}^{3}+3 c_{3}^{2}+6 c_{2} c_{4}-6 c_{6}
\end{array}\right) ; \\
& \operatorname{ch}_{7}=\frac{1}{7 !}\left(\begin{array}{l}
-9 c_{1}^{7}+7 c_{2} c_{1}^{5}+35 c_{3} c_{1}^{4}-14 c_{2}^{2} c_{1}^{3}+70 c_{4} c_{1}^{3}-42 c_{2} c_{3} c_{1}^{2} \\
+70 c_{5} c_{1}^{2}+7 c_{2}^{3} c_{1}-14 c_{3}^{2} c_{1}-28 c_{2} c_{4} c_{1} \\
+35 c_{6} c_{1}+7 c_{2}^{2} c_{3}-7 c_{3} c_{4}-7 c_{2} c_{5}+7 c_{7}
\end{array}\right) ; \\
& \operatorname{ch}_{8}=\frac{1}{8 !}\left(\begin{array}{l}
9 c_{1}^{8}-8 c_{2} c_{1}^{6}-48 c_{3} c_{1}^{5}+20 c_{2}^{2} c_{1}^{4}-120 c_{4} c_{1}^{4}+80 c_{2} c_{3} c_{1}^{3} \\
-160 c_{5} c_{1}^{3}-16 c_{2}^{3} c_{1}^{2}+40 c_{3}^{2} c_{1}^{2}+80 c_{2} c_{4} c_{1}^{2}-120 c_{6} c_{1}^{2} \\
-32 c_{2}^{2} c_{3} c_{1}+40 c_{3} c_{4} c_{1}+40 c_{2} c_{5} c_{1}-48 c_{7} c_{1}+2 c_{2}^{4} \\
-8 c_{2} c_{3}^{2}+4 c_{4}^{2}-8 c_{2}^{2} c_{4}+8 c_{3} c_{5}+8 c_{2} c_{6}-8 c_{8}
\end{array}\right) \\
& \operatorname{ch}_{9}=\frac{3}{9 !}\left(\begin{array}{l}
-3 c_{1}^{9}+3 c_{2} c_{1}^{7}+21 c_{3} c_{1}^{6}-9 c_{2}^{2} c_{1}^{5}+63 c_{4} c_{1}^{5}-45 c_{2} c_{3} c_{1}^{4} \\
+105 c_{5} c_{1}^{4}+10 c_{2}^{3} c_{1}^{3}-30 c_{3}^{2} c_{1}^{3}-60 c_{2} c_{4} c_{1}^{3}+105 c_{6} c_{1}^{3} \\
+30 c_{2}^{2} c_{3} c_{1}^{2}-45 c_{3} c_{4} c_{1}^{2}-45 c_{2} c_{5} c_{1}^{2}+63 c_{7} c_{1}^{2}-3 c_{2}^{4} c_{1} \\
+15 c_{2} c_{3}^{2} c_{1}-9 c_{4}^{2} c_{1}+15 c_{2}^{2} c_{4} c_{1}-18 c_{3} c_{5} c_{1}-18 c_{2} c_{6} c_{1} \\
+21 c_{8} c_{1}+c_{3}^{3}-3 c_{2}^{3} c_{3}+6 c_{2} c_{3} c_{4} \\
+3 c_{2}^{2} c_{5}-3 c_{4} c_{5}-3 c_{3} c_{6}-3 c_{2} c_{7}+3 c_{9}
\end{array}\right)
\end{aligned}
$$




$$
\operatorname{ch}_{10}=\frac{1}{10 !}\left(\begin{array}{l}
9 c_{1}^{10}-10 c_{2} c_{1}^{8}-80 c_{3} c_{1}^{7}+35 c_{2}^{2} c_{1}^{6}-280 c_{4} c_{1}^{6} \\
+210 c_{2} c_{3} c_{1}^{5}-560 c_{5} c_{1}^{5}-50 c_{2}^{3} c_{1}^{4}+175 c_{3}^{2} c_{1}^{4} \\
+350 c_{2} c_{4} c_{1}^{4}-700 c_{6} c_{1}^{4}-200 c_{2}^{2} c_{3} c_{1}^{3}+350 c_{3} c_{4} c_{1}^{3} \\
+350 c_{2} c_{5} c_{1}^{3}-560 c_{7} c_{1}^{3}+25 c_{2}^{4} c_{1}^{2}-150 c_{2} c_{3}^{2} c_{1}^{2} \\
+105 c_{4}^{2} c_{1}^{2}-150 c_{2}^{2} c_{4} c_{1}^{2}+210 c_{3} c_{5} c_{1}^{2}+210 c_{2} c_{6} c_{1}^{2} \\
-280 c_{8} c_{1}^{2}-20 c_{3}^{3} c_{1}+50 c_{2}^{3} c_{3} c_{1}-120 c_{2} c_{3} c_{4} c_{1} \\
-60 c_{2}^{2} c_{5} c_{1}+70 c_{4} c_{5} c_{1}+70 c_{3} c_{6} c_{1}+70 c_{2} c_{7} c_{1} \\
-80 c_{9} c_{1}-2 c_{2}^{5}+15 c_{2}^{2} c_{3}^{2}-10 c_{2} c_{4}^{2}+5 c_{5}^{2}+10 c_{2}^{3} c_{4} \\
-10 c_{3}^{2} c_{4}-20 c_{2} c_{3} c_{5}-10 c_{2}^{2} c_{6} \\
+10 c_{4} c_{6}+10 c_{3} c_{7}+10 c_{2} c_{8}-10 c_{10}
\end{array}\right) .
$$

$\operatorname{ch}\left(\Omega_{10}^{10}\right)$ :

$$
\operatorname{ch}_{k}=(-)^{k} \frac{c_{1}^{k}}{k !} \text {. }
$$

\section{References}

[1] P. Griphiths, J. Harris, Principles of Algebraic Geometry, John Wiley \& Sons, NY (1978).

[2] V.N. Dumachev, Complete intersection Calabi-Yau six-folds, Appl. Math. Sci., 9, No 143 (2015), 7121-7137; doi: 10.12988/ams.2015.510620.

[3] F. Hirzebruch, Topological Methods in Algebraic Geometry, SpringerVerlag, NY (1978).

[4] J.A. Todd, The arithmetical invariants of algebraic loci, Proc. London Math. Soc., s-2 43, No 2178 (1938), 190-225.

[5] O. Debarre, Cohomological characterizations of the complex projective space, arXiv: 1512.04321.

[6] T. Hübsch, Calabi-Yau Manifolds. A Bestiaray for Physicist, World Sci. Publ., Singapore (1994).

[7] J. Gray, A. Haupt, A. Lukas, All complete intersection Calabi-Yau four-folds, J. High Energy Phys., 1307 (2013), arXiv: 1303.1832; doi: 10.1007/JHEP07(2013)070.

[8] A. Haupt, A. Lukas, K. Stelle, M-theory on Calabi-Yau five-folds, J. High Energy Phys., 05 (2009), arXiv: 0810.2685; doi: 10.1088/1126$6708 / 2009 / 05 / 069$. 
[9] V.N. Dumachev, Seven folds of complete intersection Calabi-Yau, International J. of Pure and Applied Math., 116, No 4 (2017), 959-965; doi: 10.12732/ijpam.v116i4.12.

[10] V.N. Dumachev, Eight-folds of complete intersection Calabi-Yau, Communications in Appl. Anal., 22, No 3 (2018), 447-457; doi: 10.12732/caa.v22i3.7.

[11] V.N. Dumachev, Complete intersection Calabi-Yau nine-folds, International Journal of Applied Mathematics, 31, No 6 (2018), 853-869; DOI: 10.12732/ijam.v31i6.13. 
